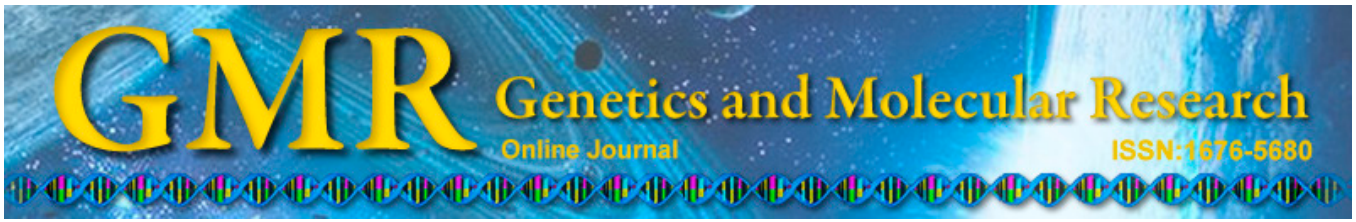

\title{
Analysis and clinical significance of microRNA-499 expression levels in serum of patients with acute myocardial infarction
}

\author{
C.H. Zhao, G.-C. Cheng, R.L. He, Y. Hong, Q.L. Wan, Z.-Z. Wang \\ and Z.-Y. Pan \\ Department of Cardiology, Henan University Huaihe Hospital, Kaifeng, \\ Henan, China \\ Corresponding author: G.-C. Cheng \\ E-mail: guanchangcheng@yeah.net
}

Genet. Mol. Res. 14 (2): 4027-4034 (2015)

Received June 25, 2014

Accepted October 29, 2014

Published April 27, 2015

DOI http://dx.doi.org/10.4238/2015.April.27.17

\begin{abstract}
The aim of this study was to investigate the expression level of microRNA-499 and its clinical significance in serum of patients with acute myocardial infarction (AMI). We recruited 59 patients with AMI and 60 healthy individuals undergoing physical examination in our hospital during the same period as controls. Peripheral blood was drawn in the morning on the same day of microRNA extraction. The expression level of microRNA-499 was analyzed by real-time fluorescent quantitative polymerase chain reaction (qPCR). The sensitivity and specificity of the clinical diagnosis of AMI were analyzed by a receiver operating characteristic (ROC) curve. Fluorescent qPCR analysis showed that the expression of microRNA-499 in serum of patients with AMI was significantly higher than in controls $(\mathrm{P}<0.05)$. MicroRNA-499 was detected in blood serum $3 \mathrm{~h}$ post-AMI, reaching a peak after $12 \mathrm{~h}$ and declining after $15 \mathrm{~h}$. The area under the ROC curve (AUC) for the gold standard cardiac troponin I (cTnI) was 0.971 [95\% confidence interval (CI): 0.951-1.000], and for the microRNA-499, AUC $=0.915$ (95\%CI: 0.826-1.000). When the microRNA-499 levels
\end{abstract}


in patient and control $(>1.5)$ sera were compared, the sensitivity of microRNA-499 in judging AMI was found to be $86.37 \%$ and the specificity was $93.47 \%$. Our results demonstrated that the expression levels of microRNA-499 in serum of patients with AMI were abnormal. Its high sensitivity and specificity for the diagnosis of AMI suggest that it would be useful as an auxiliary index for clinical diagnosis of AMI.

Key words: Acute myocardial infarction; MicroRNA-499; Sensitivity; Specificity

\section{INTRODUCTION}

Acute myocardial infarction (AMI) is a common clinical cardiovascular and cerebrovascular disease. Because of its sudden onset, rapid progress, and high death rate, it has become one of the primary diseases currently threatening human health (Lipinski et al., 2014; Velibey et al., 2014). The early discovery, diagnosis, and treatment are of great significance to the prognosis of patients with AMI. Many clinical studies have demonstrated that there are no obvious clinical symptoms during the early onset phase of AMI, and only $50 \%$ of patients have abnormal electrocardiograms (Yahalom et al., 2013). Cardiac troponin I (cTnI) is the gold standard for early clinical diagnosis of AMI. Studies showed high specificity and sensitivity of $\mathrm{cTnI}$ in the diagnosis of AMI. However, elevated cTnI expression has also been observed in patients with acute and chronic renal failure. Therefore, we need a more specific serum marker as the clinical diagnosis standard for AMI (Freund et al., 2011; Keller et al., 2011).

MicroRNA-499 is a recently discovered member of the microRNA family encoded by myosin. It has been shown to be expressed in myocardium and skeletal muscle in mammals (Rossi et al., 2010; Wang et al., 2010). Under normal physiological conditions, the expression level of microRNA-499 is low. It regulates the expression of the beta myosin heavy chain, leading to enhancement of myocardial oxygen metabolism and tolerance (Wilson et al., 2010). It has recently been demonstrated that the expression level of microRNA-499 exhibited a significant change in certain heart diseases, but there was no significant change in expression level in other heart diseases. Many studies have also shown significant levels of expression of serum microRNA-499 in AMI patients (Matkovich et al., 2009; D'Alessandra et al., 2010). Therefore, microRNA-499 might be expected to become part of the clinical auxiliary diagnostic criteria of AMI. In this study, we established a method for detection of serum microRNA-499 by fluorescence quantitative polymerase chain reaction (qPCR), and analyzed the dynamic changes of microRNA-499 in patients with AMI and its sensitivity and specificity as a clinical diagnostic marker, with the aim to provide a new diagnostic marker for clinical diagnosis of AMI.

\section{MATERIAL AND METHODS}

\section{General subject data}

Of the patients with AMI admitted in our hospital from June 2010 to June 2013, 59 were chosen for this study. Inclusion criteria for this study were: 1) diagnosis according to the World Health Organization clinical diagnosis criteria for AMI (Alpert et al., 2000); 2) no 
mechanical trauma, liver and kidney function damage, thyroid hyperfunction or hypofunction, tumor, immune dysfunction, or other diseases; 3 ) patients were in first onset and the time from onset to hospitalization was no more than $24 \mathrm{~h} ; 4$ ) no previous diagnosis of diabetes, hypertension, cerebral infarction, etc.; 5) patients provided signed informed consent. There were 34 men and 25 women in the observation group. The age range was 54-76 years, and the average age was $60.1 \pm 11.3$ years. With respect to the infarction site, there were 20 cases of anterior wall, 13 cases of inferior wall, 20 cases of anterior wall, and 6 cases of inferior wall and right ventricular myocardial infarction. Concurrently, we chose 60 healthy volunteers as a control group, including 30 men and 30 women. The age range of the control group was 52-69 years, and the average age was $61.9 \pm 12.1$ years. There was no significant difference in age and gender between the two groups $(\mathrm{P}>0.05)$. This study was conducted in accordance with the Declaration of Helsinki, and with approval from the Ethics Committee of Henan University Huaihe Hospital. Written informed consent was obtained from all participants.

\section{Sample collection and extraction of serum total RNA}

We collected $5 \mathrm{~mL}$ fasting venous blood from subjects in the observation and control groups in the morning, and samples were processed for RNA extraction on the same day according to the following protocol. Samples were placed at room temperature for $3 \mathrm{~h}$, centrifuged at $3000 \mathrm{rpm}$ for $15 \mathrm{~min}$ and the serum removed. Samples were re-centrifuged at $12,000 \mathrm{rpm}$ for $15 \mathrm{~min}$ at $4^{\circ} \mathrm{C}$, and the liquid supernatant was withdrawn. RNA extraction was performed using the TRI reagent $\mathrm{BD}$ kit according to manufacturer instructions as follows: $200 \mu \mathrm{L}$ plasma was added to a $1.5 \mathrm{~mL}$ centrifuge tube containing $750 \mu \mathrm{L}$ TRI reagent $\mathrm{BD}$, followed by addition of $20 \mu \mathrm{L} 5 \mathrm{~N}$ glacial acetic acid. Tubes were reverse mixed, $5 \mu \mathrm{L} 50 \mathrm{pM}$ Caenorhabditis elegans miRNA (ce-miR-39) was added, and the tubes were mixed again and stored at room temperature for $10 \mathrm{~min}$ prior to addition of $200 \mu \mathrm{L}$ chloroform followed by a second rest at room temperature for $5 \mathrm{~min}$. Tubes were centrifuged at 12,000 rpm for $15 \mathrm{~min}$ and the supernatant was extracted. DNAmate $(2 \mu \mathrm{L})$ was added, and the tubes were blended followed by addition of an equal volume of isopropanol and stored overnight at $-80^{\circ} \mathrm{C}$. The next day, samples were centrifuged at $12,000 \mathrm{rpm}$ for $15 \mathrm{~min}$, and the supernatant was discarded. Precipitation was used by anhydrous ethanol prepared by precooling $75 \%$ DEPC water to wash, and centrifuged at $8000 \mathrm{rpm}$ for $5 \mathrm{~min}$. The supernatant was discarded, and residual ethanol was dried in the fume hood. DEPC-treated distilled water was added $(30 \mu \mathrm{L})$ to completely dissolve the RNA precipitate. The concentration and purity of the total RNA was determined by spectrophotometry.

\section{Establishment of the fluorescent qPCR system}

Total RNA $(1 \mu \mathrm{g})$ was transcribed into cDNA using a reverse transcription kit (TaKaRa; Dalian, China) with the following reaction mixture: $2 \mu \mathrm{L} 5 *$ PrimeScript Buffer, $0.5 \mu \mathrm{L}$ PrimerScript RT Enzyme mix, $0.5 \mu \mathrm{L}$ Oligo dT primer $(50 \mu \mathrm{M}), 2 \mu \mathrm{L}$ random hexamer primer $(100 \mu \mathrm{M}), 0.5 \mu \mathrm{L}$ stem-loop RT primer, $2 \mu \mathrm{L}$ total RNA, and $2.5 \mu \mathrm{L}$ RNase Free $\mathrm{dH}_{2} \mathrm{O}$. The reaction mixture was placed at $42^{\circ} \mathrm{C}$ for $30 \mathrm{~min}$, then inactivated at $85^{\circ} \mathrm{C}$ and stored at $4^{\circ} \mathrm{C}$.

The qPCR mixture was prepared as follows: $10 \mu \mathrm{L} 2 *$ SYBR Green general qPCR Master Mix (Roche, Basel, Switzerland), $1 \mu \mathrm{L}$ of each upstream and downstream primer (10 $\mu \mathrm{M}), 1 \mu \mathrm{L}$ cDNA, complemented with double-distilled water to a final volume of $20 \mu \mathrm{L}$. Ac- 
cording to the number of samples, we prepared the corresponding volume of reaction mix, and placed $20 \mu \mathrm{L}$ into each well of a PCR plate, followed by centrifugation at $1500 \mathrm{rpm}$ to ensure that the reaction mixture was at the bottom of the well. PCR was performed according to the following reaction conditions: predenaturation at $95^{\circ} \mathrm{C}$ for $30 \mathrm{~s}$, denaturation at $95^{\circ} \mathrm{C}$ for 3 $\mathrm{s}$, annealing and extension at $60^{\circ} \mathrm{C}$ for $30 \mathrm{~s}$, followed by generation of a melting curve. Data were obtained directly from the real-time fluorescence quantitative PCR instrument, utilizing the amplification profile of cel-miR-39 as an internal standard.

\section{Detection of cTnI}

Serum cTnI was detected by chemiluminescence. The detection method was performed according to the manufacturer instructions (Roche). The level of cTnI $>0.08 \mathrm{ng} / \mathrm{mL}$ was used as a positive standard.

\section{Observation indices}

The microRNA-499 levels in serum in the control group and the observation group were analyzed, and dynamic changes of microRNA-499 in the serum in the observation group were determined. The sensitivity and specificity of microRNA-499 in the clinical diagnosis of AMI were determined.

\section{Statistical analysis}

All data were analyzed using the SPSS13.0 statistical software (SPSS Inc., Chicago, IL, USA). The measurement data are reported as means $\pm \mathrm{SD}$. The measurement data between groups were compared by the Student $t$-test. The receiver operator characteristic (ROC) curve was drawn using the GraphPad 5 software (GraphPad, LaJolla, CA, USA). We calculated the cut-off value, and the sensitivity, and specificity. $\mathrm{P}<0.05$ indicated that the difference was considered to be statistically significant.

\section{RESULTS}

\section{Two-group comparison of serum microRNA-499 levels}

The average serum microRNA-499 level in the control group was $0.70 \pm 0.15$ and that in the observation group was $22.10 \pm 3.71$. The serum microRNA-499 level in the observation group was significantly higher than that in the control group $(\mathrm{P}<0.05)$ (Figure 1).

\section{Changes of microRNA-499 levels in patients at different time points}

The real-time dynamic observation of serum microRNA-499 in 12 patients was performed from 0 to $24 \mathrm{~h}$ after the onset of AMI. MicroRNA-499 could be detected in the serum $3 \mathrm{~h}$ after the onset of AMI, reached a peak value after $12 \mathrm{~h}$, and gradually declined after $15 \mathrm{~h}$ (Figure 2). This result indicated that following microRNA-499 induction, the miRNA could be rapidly released into the blood. This indicates that microRNA-499 may be helpful to clinical diagnosis and detection. 


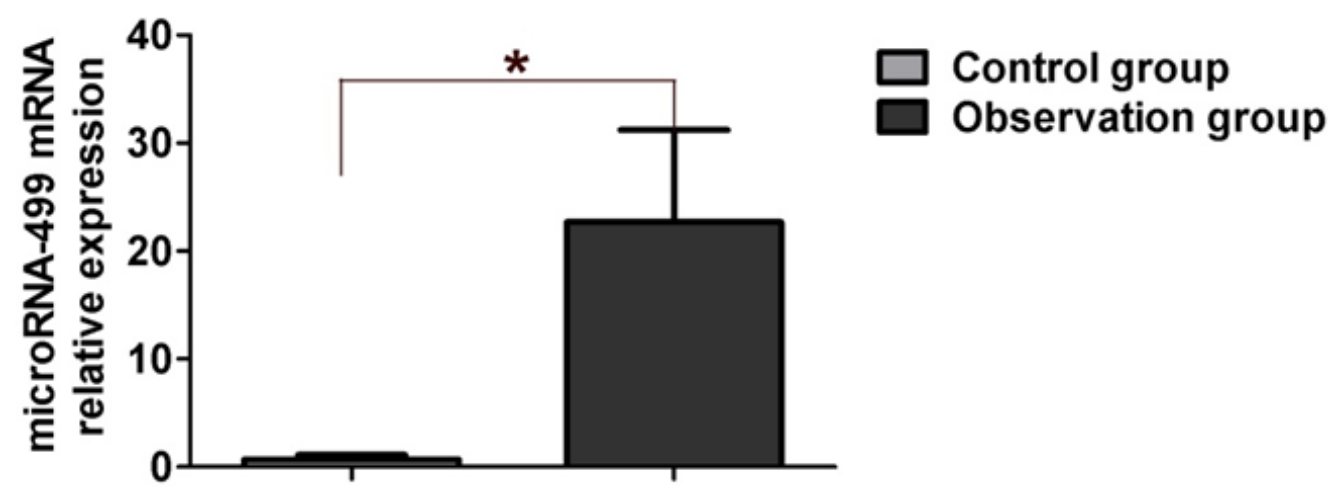

Figure 1. Comparison of serum microRNA-499 level in control group and observation group.

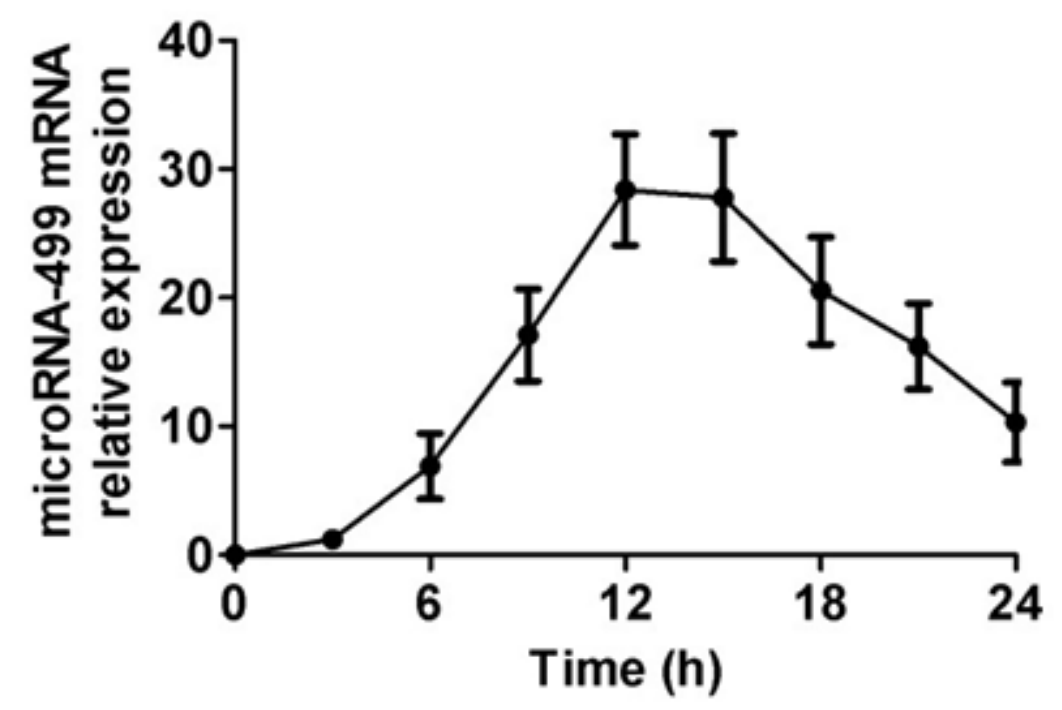

Figure 2. Dynamic changes of microRNA-499 levels in AMI patients of the observation group.

\section{Level of microRNA-499 in patients with different diseases}

In order to determine whether it also improved significantly in other diseases except for the abnormal expression of serum microRNA-499 in AMI patients. This study compared the serum microRNA-499 level in patients with stroke, acute and chronic renal failure, and trauma. As shown in Figure 3, the expression level of serum microRNA-499 in patients with stroke, acute and chronic kidney failure, or trauma was low, at a level equivalent with that in the healthy patients. This indicated that there was a high specificity of the phenomenon of release of serum microRNA-499 to patients with AMI as opposed to other disorders, and it is expected to become a marker for the diagnosis of AMI. 


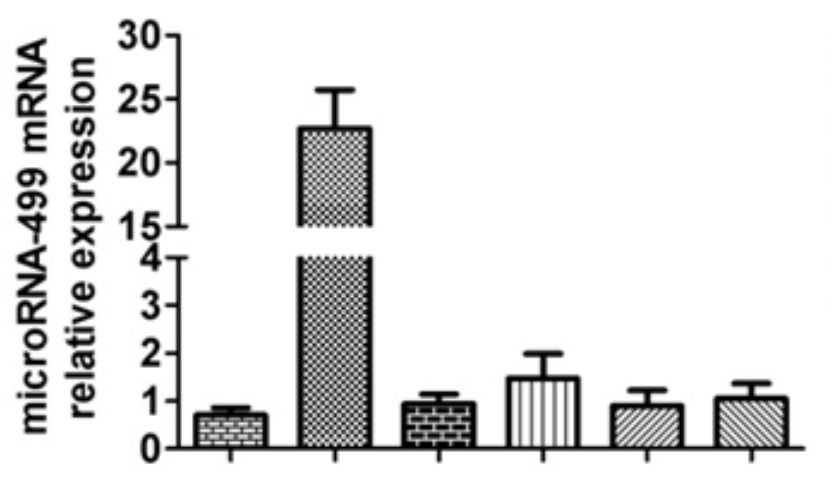

\section{国 Control group Observation group \\ 四 Stroke \\ 血 Acute renal failure Chronic renal failure Traumatic}

Figure 3. Comparison of microRNA-499 in different disease patients.

\section{Analysis of the sensitivity and specificity of microRNA-499}

Following our observation of the abnormal expression of microRNA-499 in the serum of patients with AMI, we analyzed whether this feature could be used as a diagnostic marker of AMI by using an ROC curve. Taking $\mathrm{cTnI}$ as the gold standard for AMI diagnosis, the area under the ROC curve (AUC) for cTnI was 0.971 [95\% confidence interval (CI): 0.951-1.000] (Figure 4). When the level of cTnI was $>0.08 \mathrm{ng} / \mathrm{mL}$, the sensitivity of diagnosis of AMI was $93.12 \%$ and the specificity was $100 \%$. The AUC of microRNA-499 was 0.915 (95\%CI: $0.826-$ 1.000). When the serum microRNA-499 level of AMI patients was compared with normal levels $(>1.5 \mathrm{ng} / \mathrm{mL})$, the sensitivity of microRNA-499 in judging AMI was $86.37 \%$ and the specificity was $93.47 \%$. These results indicated that the sensitivity and specificity of microRNA-499 in the diagnosis of AMI were lower than those of cTnI, but that it still has the potential to be used as a clinical assistant or joint diagnostic marker.

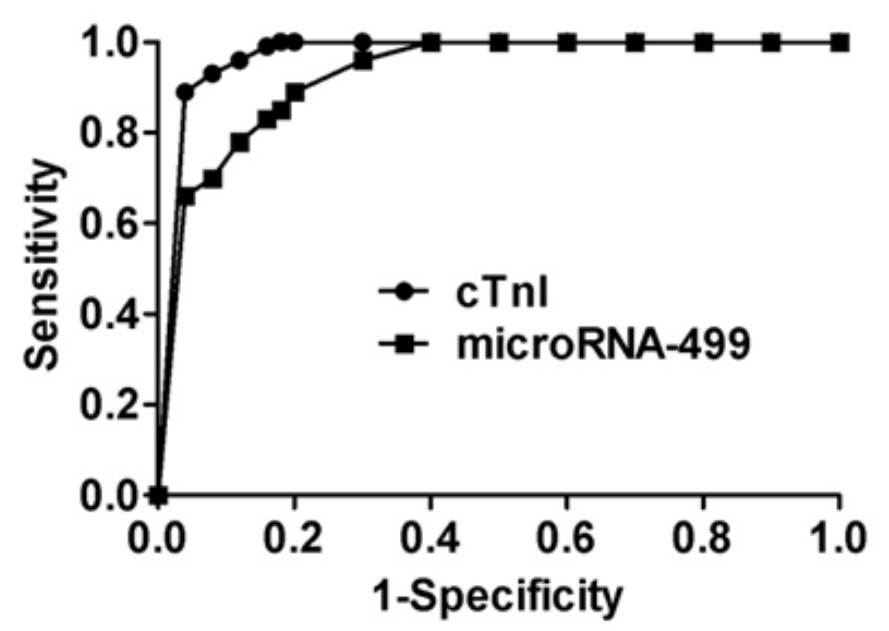

Figure 4. Sensitivity and specificity analysis by microRNA-499 in AMI patients. 


\section{DISCUSSION}

There are currently many markers available for the clinical diagnosis of AMI, including creatine kinase (CK), creatine kinase isoenzyme (CK-MB), cTnI, and myoglobin (MYO). (Chon et al., 2014; Kruse et al., 2014). A clinical diagnostic marker must have high tissue specificity and sensitivity on the one hand, and must be rapidly released into the bloodstream after myocardial damage on the other hand, and must have a short half-life. MYO was the earliest diagnostic marker identified for AMI. Its sensitivity was high, but it exhibited low tissue specificity (Storrow and Gibler, 1999). CK and CK-MB also decreased due to tissue specificity. Therefore, cTnI became the gold standard for clinical diagnosis of AMI; its outstanding advantage in the diagnosis of AMI was its high specificity. cTnI shows great potential for the prognosis, risk stratification, and treatment of AMI (Apple and Murakami, 2005). However the half-life of cTnI is relatively long, so it could not be used for the diagnosis of re-infarction after initial myocardial infarction occurrence. Furthermore, its expression levels in patients with sepsis or nephropathy are often elevated. It has therefore become necessary to identify a clinical diagnosis marker for AMI that exhibits both high specificity and sensitivity (HowieEsquivel and White, 2008).

MicroRNAs are highly conserved non-coding small RNAs that can degrade target mRNA or interfere in its translation by pairing with mRNA completely or partially (Salic and De Windt, 2012). Recent studies have found that microRNA expression profiles were significantly changed in the process of some diseases. This indicated that microRNAs play an important role in the pathophysiology of disease (Ai et al., 2010). At present, numerous microRNAs have already been found in serum through the application of high-throughput sequencing, and these are widely used in clinical diagnosis and in the determination of prognosis of tumors and other diseases (Ganepola et al., 2014).

MicroRNA-499 is a recently discovered microRNA, highly conserved in mammals, and primarily shown to exist in the myocardium and skeletal muscle. Many studies have found that the plasma levels of microRNA-499 were significantly increased in patients with AMI (Matkovich et al., 2009; Shieh et al., 2011). Our study also confirmed that the plasma levels of microRNA-499 in patients with AMI were significantly higher than those in healthy individuals. The plasma half-life of microRNA-499 is short, allowing us to determine that its levels significantly increased $3 \mathrm{~h}$ after the onset of AMI, reached a peak value at $12 \mathrm{~h}$, and then declined gradually. We expect that this phenomenon would be helpful to clinical diagnosis of re-infarction after an initial AMI has already occurred. Therefore, microRNA-499 is expected to become a clinical marker for the diagnosis of AMI.

Because the specificity of $\mathrm{cTnI}$ in renal failure patients is low, we analyzed the changes of micrRNA-499 in patients with such diseases to see whether it also might be non-specific. Our study showed that the levels of microRNA-499 in stroke, acute and chronic renal failure, and trauma patients was equivalent with that in healthy individuals, indicating that utilization of microRNA-499 might make up for the deficiency of cTnI in clinical diagnosis. Regarding cTnI as the gold standard, we analyzed the specificity and sensitivity of microRNA-499 as a marker of AMI. The results showed that when the microRNA-499 level in the serum of patients with AMI was compared to levels in normal individuals $(>1.5 \mathrm{ng} / \mathrm{mL})$, the sensitivity of microRNA-499 in judging AMI was $86.37 \%$ and the specificity was $93.47 \%$. The results showed that the specificity and sensitivity of microRNA-499 in the diagnosis of AMI were still lower than those of cTnI, and our study showed that microRNA-499 can make up for 
the deficiency of cTnI. Therefore, microRNA-499 could be used as an auxiliary index for the clinical diagnosis of AMI and, in combination with cTnI, could increase the accuracy of clinical diagnosis.

In conclusion, the expression level of microRNA-499 in AMI patients was significantly increased, and its clinical diagnostic specificity and sensitivity were determined to be slightly lower than those of cTnI. However, it could make up for the deficiency of the diagnosis of cTnI, and might be useful as an auxiliary index for the clinical diagnosis of AMI.

\section{REFERENCES}

Ai J, Zhang R, Li Y, Pu J, et al. (2010). Circulating microRNA-1 as a potential novel biomarker for acute myocardial infarction. Biochem. Biophys. Res. Commun. 391: 73-77.

Alpert JS, Thygesen K, Antman E and Bassand JP (2000). Myocardial infarction redefined - a consensus document of The Joint European Society of Cardiology/American College of Cardiology Committee for the redefinition of myocardial infarction. J. Am. Coll. Cardiol. 36: 959-969.

Apple FS and Murakami MM (2005). Cardiac troponin and creatine kinase MB monitoring during in-hospital myocardial reinfarction. Clin. Chem. 51: 460-463.

Chon H, Lee S, Yoon SY, Lee EK, et al. (2014). SERS-based competitive immunoassay of troponin I and CK-MB markers for early diagnosis of acute myocardial infarction. Chem. Commun. (Camb.) 50: 1058-1060.

D'Alessandra Y, Devanna P, Limana F, Straino S, et al. (2010). Circulating microRNAs are new and sensitive biomarkers of myocardial infarction. Eur. Heart J. 31: 2765-2773.

Freund Y, Chenevier-Gobeaux C, Bonnet P, Claessens YE, et al. (2011). High-sensitivity versus conventional troponin in the emergency department for the diagnosis of acute myocardial infarction. Crit. Care 15: R147.

Ganepola GA, Nizin J, Rutledge JR and Chang DH (2014). Use of blood-based biomarkers for early diagnosis and surveillance of colorectal cancer. World J. Gastrointest. Oncol. 6: 83-97.

Howie-Esquivel J and White M (2008). Biomarkers in acute cardiovascular disease. J. Cardiovasc. Nurs. 23: 124-131.

Keller T, Zeller T, Ojeda F, Tzikas S, et al. (2011). Serial changes in highly sensitive troponin I assay and early diagnosis of myocardial infarction. JAMA 306: 2684-2493.

Kruse JM, Enghard P, Schröder T, Hasper D, et al. (2014). Weak diagnostic performance of troponin, creatine kinase and creatine kinase-MB to diagnose or exclude myocardial infarction after successful resuscitation. Int. J. Cardiol. 173: 216-221.

Lipinski MJ, Escárcega RO, D’Ascenzo F, Magalhães MA, et al. (2014). A systematic review and collaborative metaanalysis to determine the incremental value of copeptin for rapid rule-out of acute myocardial infarction. Am. $J$. Cardiol. 113: 1581-1591.

Matkovich SJ, Van Booven DJ, Youker KA, Torre-Amione G, et al. (2009). Reciprocal regulation of myocardial microRNAs and messenger RNA in human cardiomyopathy and reversal of the microRNA signature by biomechanical support. Circulation 119: 1263-1271.

Rossi AC, Mammucari C, Argentini C, Reggiani C, et al. (2010). Two novel/ancient myosins in mammalian skeletal muscles: MYH14/7b and MYH15 are expressed in extraocular muscles and muscle spindles. J. Physiol. 588: 353-364.

Salic K and De Windt LJ (2012). MicroRNAs as biomarkers for myocardial infarction. Curr. Atheroscler. Rep. 14: 193200.

Shieh JT, Huang Y, Gilmore J and Srivastava D (2011). Elevated miR-499 levels blunt the cardiac stress response. PLoS One 6: e19481.

Storrow AB and Gibler WB (1999). The role of cardiac markers in the emergency department. Clin. Chim. Acta 284: 187-196.

Velibey Y, Erbay A, Ozkurt E, Usta E, et al. (2014). Acute myocardial infarction associated with blood transfusion: case report and literature review. Transfus. Apher. Sci. 50: 260-262.

Wang GK, Zhu JQ, Zhang JT, Li Q, et al. (2010). Circulating microRNA: a novel potential biomarker for early diagnosis of acute myocardial infarction in humans. Eur. Heart J. 31: 659-666.

Wilson KD, Hu S, Venkatasubrahmanyam S, Fu JD, et al. (2010). Dynamic microRNA expression programs during cardiac differentiation of human embryonic stem cells: role for miR-499. Circ. Cardiovasc. Genet. 3: 426-435.

Yahalom M, Roguin N, Suleiman K and Turgeman Y (2013). Clinical significance of conditions presenting with ECG changes mimicking acute myocardial infarction. Int. J. Angiol. 22: 115-122. 